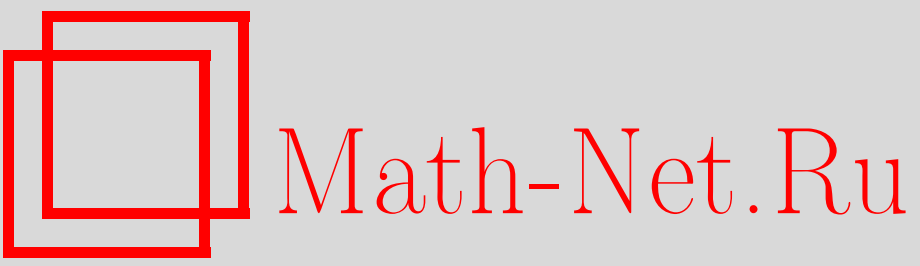

О. В. Шатаев, Минимизация относительной энтропии в задаче нахождения мартингальной меры, УМH, 2000, том 55, выпуск 5, 183-184

DOI: https://doi.org/10.4213/rm330

Использование Общероссийского математического портала Math-Net.Ru подразумевает, что вы прочитали и согласны с пользовательским соглашением

http://www . mathnet.ru/rus/agreement

Параметры загрузки:

IP : 54.84 .234 .179

26 апреля 2023 г., 15:48:53 


\title{
МИНИМИЗАЦИЯ ОТНОСИТЕЛЬНОЙ ЭНТРОПИИ В ЗАДАЧЕ НАХОЖДЕНИЯ МАРТИНГАЛЬНОЙ МЕРЫ
}

\author{
O. В. ШАТАЕВ
}

Пусть $(\Omega, \mathscr{F}, \mathrm{P})$ - исходное вероятностное пространство, $h=\left(h_{n}\right)_{1 \leqslant n \leqslant N}$ - действительнозначные случайные величины, имеющие непрерывное совместное распределение со всюду ненулевой плотностью $f_{0}\left(x_{1}, \ldots, x_{N}\right)>0$.

Через $\mathscr{F}_{n}$ обозначаем $\sigma$-алгебры $\sigma\left(h_{1}, \ldots, h_{n}\right), \mathscr{F}_{0}=\{\varnothing, \Omega\}$.

Будем интерпретировать $S_{n}=S_{0} e^{h_{1}+\cdots+h_{n}}$ как значение цены акции в момент времени $n=1, \ldots, N$, а $B_{n} \equiv 1-$ как цену облигации. Обозначим $\mathscr{P}(\mathrm{P})$ - множество всех эквивалентных исходной мере $\mathrm{P}$ вероятностных мер таких, что $\left(\frac{S_{n}}{B_{n}}\right)$ - мартингал относительно фильтрации $\mathscr{F}=\left(\mathscr{F}_{n}\right)$. Меры $\widetilde{\mathrm{P}} \in \mathscr{P}(\mathrm{P})$ будем называть мартингальными.

Мы рассматриваем проблему нахождения плотности $f\left(x_{1}, \ldots, x_{N}\right)$ совместного распределения $h_{1}, \ldots, h_{N}$, соответсвующего мартингальной мере $\widetilde{\mathrm{P}} \in \mathscr{P}(\mathrm{P})$, удовлетворяющей условиям

$$
\mathrm{E}_{\widetilde{\mathrm{P}}} G_{i}\left(S_{1}, \ldots, S_{N}\right)=\int G_{i} f d x_{1} \cdots d x_{N}=C_{i}, \quad 1 \leqslant i \leqslant M
$$

где $G_{i}$ - заданные функции, интерпретируемые как дисконтированные платежные функции некоторых опционов, а $C_{i}$ - заданные значения их цен на момент времени 0.

Исходя из условий (1) плотность $f$ определена неоднозначно. Поэтому, следуя [10], выберем плотность, минимизирующую расстояние Кульбака-Лейблера (относительную энтропию)

$$
H\left(f \mid f_{0}\right)=\int f \log \left(\frac{f}{f_{0}}\right) d x_{1} \cdots d x_{N}
$$

где $f_{0}$ - априорная плотность вероятностного распределения.

Учитывая, что условия мартингальности $\left(\frac{S_{n}}{B_{n}}\right)$ в терминах плотности распределения принимают вид

$$
\int\left(e^{x_{i+1}}-1\right) f\left(h_{1}, \ldots, h_{i}, x_{i+1}, \ldots, x_{N}\right) d x_{i+1} \cdots d x_{N}=0 \text { Р-п.н., }
$$

а функционал $H\left(f \mid f_{0}\right)$ выпукл по $f$, заметим, что мы решаем задачу минимизации выпуклого ограниченного снизу функционала при линейных ограничениях. Таким образом, по теореме Куна-Таккера о седловой точке наша задача эквивалентна нахождению

$$
\begin{aligned}
\inf _{\lambda_{i}, p_{j}} \inf _{\mu} \sup _{f}\left[-H\left(f \mid f_{0}\right)+\sum_{j} \int p_{j}\left(x_{1}, \ldots, x_{j}\right)\left(e^{x_{j}+1}-1\right) f d x_{1} \cdots d x_{N}\right. \\
\left.\quad+\sum_{i} \lambda_{i}\left(\int G_{i} f d x_{1} \cdots d x_{N}-C_{i}\right)+\mu\left(\int f d x_{1} \cdots d x_{N}-1\right)\right] .
\end{aligned}
$$

Для начала, зафиксировав $\lambda, p$ и $\mu$, найдем методами вариационного исчисления функцию $f_{\lambda, p, \mu}$, максимизирующую лагранжиан: $f_{\lambda, p, \mu}=f_{0} \exp \left\{-1+\mu+\sum_{j} p_{j}\left(e^{x_{j+1}}-1\right)+\sum_{i} \lambda_{i} G_{i}\right\}$. Затем подставим в лагранжиан $f_{\lambda, p, \mu}$ и определим плотность $f_{\lambda, p}$, минимизирующую полученное выражение по $\mu$ :

$$
f_{\lambda, p}=\frac{1}{Z(\lambda, p)} f_{0} e^{\sum_{j} p_{j}\left(e^{x_{j}+1}-1\right)+\sum_{i} \lambda_{i} G_{i}}
$$

где $Z(\lambda, p)$ - нормализующий множитель

$$
Z(\lambda, p)=\int f_{0} e^{\sum_{j} p_{j}\left(e^{x_{j}+1}-1\right)+\sum_{i} \lambda_{i} G_{i}} d x_{1} \cdots d x_{N} .
$$


Подставляя (5) в (4), находим, что оптимизация методом множителей Лагранжа эквивалентна минимизации по $p$ и $\lambda$ выражения

$$
\log (Z(\lambda, p))-\sum_{i} \lambda_{i} C_{i}
$$

по всем значениям $\lambda=\left(\lambda_{1}, \ldots, \lambda_{M}\right), p_{0}$ и всем ограниченным измеримым функциям $p_{1}\left(x_{1}\right), \ldots$, $p_{N-1}\left(x_{1}, \ldots, x_{N-1}\right)$. Условия первого порядка на минимум:

$$
\text { (8) } \int\left(e^{x_{k+1}}-1\right) f_{0} e^{\sum_{j=k}^{N-1} p_{j}\left(e^{x_{j}+1}-1\right)+\sum_{i} \lambda_{i} G_{i}} d x_{k+1} \cdots d x_{N} \equiv 0, \quad k=0, \ldots, N-1 \text {. }
$$

Интересно, что если при фиккированном $\lambda$ вычислять $p_{k}$, начиная с $k=N-1$ и заканчивая $k=0$, то на каждом $k$-м шаге вычислений при фиксированных $x_{1}, \ldots, x_{k}$ значение $p_{k}\left(x_{1}, \ldots, x_{k}\right)$ может быть найдено как скалярная переменная из уравнения с одним неизвестным.

Например, в случае одношаговой модели при экспоненциально распределенном $e^{h_{1}}$ с параметром 1 и при условии $S_{0}=K$ для опциона европейского типа с платежной функцией $G_{1}=$ $\left(K-S_{1}\right)^{+}$уравнения $(7)$ и (8) принимают вид:

$$
\begin{gathered}
-\frac{S_{0} e^{-1}}{\left(p_{0}-1-S_{0} \lambda_{1}\right)^{2}}+\frac{S_{0} e^{S_{0} \lambda_{1}-p_{0}}}{\left(p_{0}-1-S_{0} \lambda_{1}\right)^{2}}+\frac{S_{0} e^{S_{0} \lambda_{1}-p_{0}}}{p_{0}-1-S_{0} \lambda_{1}} \\
e^{-1}\left(\frac{1}{p_{0}-1-S_{0} \lambda_{1}}-\frac{1}{p_{0}-1}\right)-\frac{e^{S_{0} \lambda_{1}-p_{0}}}{p_{0}-1-S_{0} \lambda_{1}} \\
\frac{\left(p_{0}-S_{0} \lambda_{1}\right) e^{S_{0} \lambda_{1}-p_{0}}-e^{-1}}{\left(p_{0}-1-S_{0} \lambda_{1}\right)^{2}}+\frac{e^{-1}}{\left(p_{0}-1\right)^{2}}=0 .
\end{gathered}
$$

Суммируем резуљтаты этой работы в виде следующего утверждения.

Теорема. Плотность $f_{\lambda, p}$ распределения, соответствующего минимизирующей относительную әнтропию мартингальной мере, является представителем бесконечномерного параметрического семейства, заданного выражением (5). Причем параметры удовлетворяют уравнениям (7) и (8).

\section{СПИСОК ЛИТЕРАТУРЫ}

[1] Алексеев В. М., Тихомиров В. М., Фомин С. В. Оптимальное управление. М.: Наука, 1979. [2] Мельников А. В. Финансовые рынки. Стохастический анализ и расчет производных ценных бумаг. М.: Т.В.П., 1997. [3] Рокафеллар Р. Т. Выпуклый анализ. М.: Мир, 1973. [4] Ширяев А. Н. Вероятность. М.: Наука, 1989. [5] Ширяев А. Н. // Теория вероятн. и ее примен. 1994. Т. 39. № 1. С. 5-22. [6] Ширяев А. Н. Основы стохастической финансовой математики. Т. 1, 2. М.: ФАЗИС, 1998. [7] Ширяев А. Н., Кабанов Ю. М., Крамков Д. О., Мельников А. В. // Теория вероятн. и ее примен. 1994. Т. 39. № 1. С. 23-79. [8] Avellaneda M. The Minimum-Entropy Algorithm and Related Methods for Calibrating Asset-Pricing Model // Preprint, 1998. P. 51-73. [9] Bollerslev T. // J. Econometrics. 1986. № 31. P. 307-327. [10] Buchen P. W., Kelly M. // J. Finacial Quantitative Anal.. V. 31. № 1. [11] Duan J. // Math. Finance. 1995. № 5. P. 13-32. [12] Rockafellar R. T. Convex Analysis. Princeton: Princeton Univ. Press, 1970. 